\title{
Advanced Trajectory Generator for Two Carts with RGB-D Sensor on Circular Rail
}

\author{
Ramón Panduro ${ }^{1}$, Eva Segura ${ }^{2}$, Lidia M. Belmonte ${ }^{1,2}$, Paulo Novais ${ }^{3}$, \\ Jesús Benet ${ }^{2}$, Antonio Fernández-Caballero ${ }^{1,2}$, and Rafael Morales ${ }^{1,2}$ \\ ${ }^{1}$ Universidad de Castilla-La Mancha \\ Instituto de Investigación en Informática de Albacete, 02071-Albacete, Spain \\ ${ }^{2}$ Universidad de Castilla-La Mancha \\ Escuela Técnica Superior de Ingenieros Industriales, 02071-Albacete, Spain \\ ${ }^{3}$ Universidade do Minho, Intelligent Systems Lab, Campus of Gualtar \\ 4710-057 Braga, Portugal
}

\begin{abstract}
This paper presents a motorised circular rail that generates the motion of two carts with an RGB-D sensor each. The objective of both carts' trajectory generation is to track a person's physical rehabilitation exercises from two points of view and his/her emotional state from one of these viewpoints. The person is moving freely his/her position and posture within the circle drawn by the motorised rail. More specifically, this paper describes the calculation of trajectories for safe motion of the two carts on the motorised circular rail in detail. Lastly, a study case is offered to show the performance of the described control algorithms for trajectory generation.
\end{abstract}

Keywords: Physical rehabilitation - Facial emotion detection · Moving cart · Motorised circular rail · RGB-D sensor.

\section{Introduction}

Popularity of computer-based physical rehabilitation systems is constantly increasing. Such systems typically use depth cameras to detect and track humans [1-4]. Moreover, some previous works that provide solutions based on RGB-D for monitoring rehabilitation exercises have been presented in the last years [5, $6]$. In addition, facial emotion detection $[7,8]$ of the human doing exercises is a good way to understand how he/she feels during the rehabilitation program. Our vision-based solutions are based on human detection $[9,10]$ and tracking [11-13]. This paper is also inspired in previous research in multi-robotics [14-16] tracking robotics [17-19] and rehabilitation robotics [20-22].

It is mandatory to continuously provide excellent viewpoints by modifying the camera's angles [23] to monitor people who are undergoing physical rehabilitation programs. RGB-D sensors must be placed in the best positions in an intelligent manner to acquire images of the most relevant parts of the patients' bodies. This is why, mechanical solutions and control strategies are being developed for optimally placing RGB-D sensors during human rehabilitation exercises 


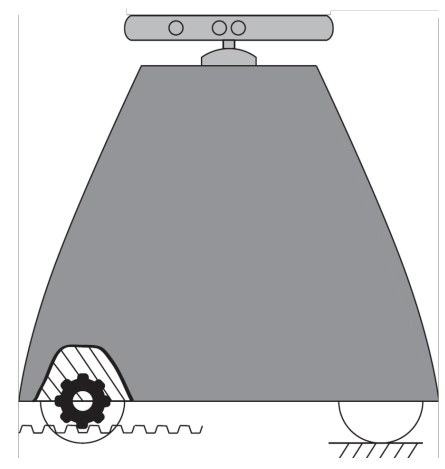

Fig. 1. Layout of a cart equipped with an RGB-D sensor.

[24]. Moreover, facial emotion detection determines that one of the viewpoints must be frontal to the patient.

This article describes an advanced trajectory generator for a motorised circular rail capable of smartly relocating two carts equipped with an RGB-D sensor each. The layout of such cart is shown in Fig. 1. The aim is to monitor a patient's face and body from two complementary views [25], one of them being frontal to the patient. In this particular design, a patient can move freely within the rail's circle, so that the carts are placed around him/her to monitor all body gestures from two sights.

\section{Trajectory Generator}

The proposed trajectory generator must calculate the displacements of both carts to locate them in the optimal position to track the patient's face and physical rehabilitation exercises. For this sake, dynamically one of the carts is assigned the role of master, in front of his/her face, and the other one the role of slave. The patient is described as input vector within the trajectory generation system. It is assumed that both the patient's tracking position and the position of the two carts are known in each instant. Several parameters are considered to correctly carry out the trajectory. These are the tilt and pan movements of the cameras (one per cart), the final angular positions of the carts with respect to the rail, the direction that each cart must follow, and the possible collisions between both carts.

\subsection{Angles of the Cameras}

First, the location of the patient is defined with respect to the circle's centre $\left(x_{p}, y_{p}\right)$ and the camera's pan angle $\gamma_{c}$. On the other hand, the camera's tilt angle $\alpha_{c}$ is defined by considering the height of the tracked zone. Fig. 2 shows an outline of this arrangement. 

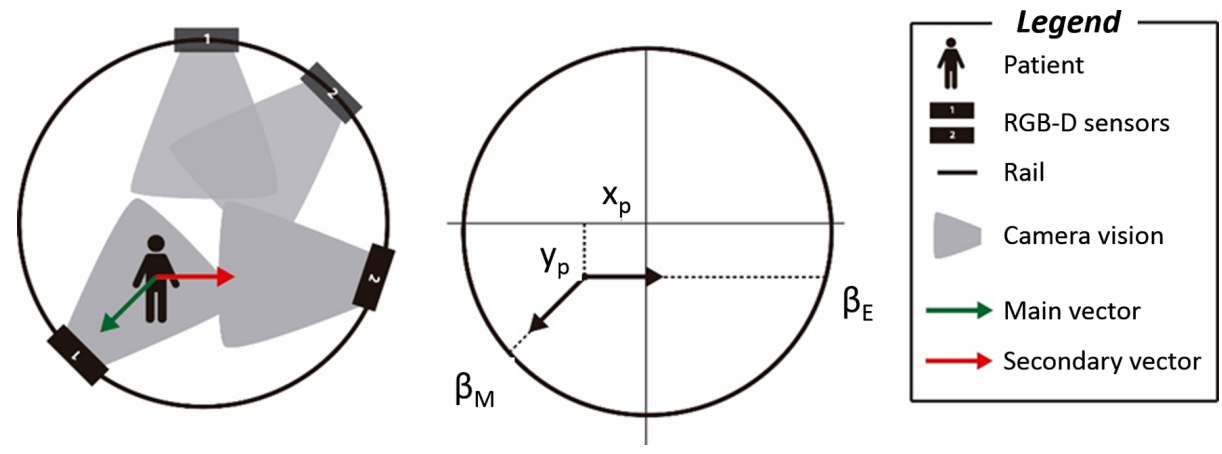

Fig. 2. Vector schemes.

The formulas that govern the behaviour of the carts are different depending on the master and slave roles assigned. These roles are established according to the current locations of both carts. On the other hand, the angular position of the slave cart must vary when a collision is foreseen with the master cart after the trajectories have been calculated. In this case, the slave cart must follow the opposite path.

In this arrangement, also the camera's pan movement $\gamma_{c}$ is calculated as follows. The direction opposite to the RGB-D sensor vector, which angle is $\gamma_{c}+$ $\pi$, moves until occupying the position of RGB-D sensor vector with angle $\gamma_{p}$. However, as the RGB-D sensor is mounted on a cart rotating around the rail, the turn $\beta_{c}$ done by the cart is subtracted, obtaining

$$
\begin{gathered}
\Delta \gamma_{1}=\gamma_{c}-\left(\gamma_{p}+\pi\right)-\min \left(\left|\Delta \beta_{1}\right|,\left|\Delta \beta_{2}\right|\right) ; \\
\Delta \gamma_{2}=\pi-\gamma_{c}+\gamma_{p}-\min \left(\left|\Delta \beta_{1}\right|,\left|\Delta \beta_{2}\right|\right)
\end{gathered}
$$

where $\Delta \beta_{1}$ and $\Delta \beta_{2}$ are magnitudes that will be defined in Section 2.2. When calculating the tilt movement of the RGB-D sensor, the height of the tracked zone $z_{p}$ is considered. The angles of the patient's vector covered by the master and slave carts, named $\alpha_{p m}$ and $\alpha_{p e}$ respectively, are calculated with respect to the RGB-D sensor height $z_{c}$. That is,

$$
\begin{aligned}
\alpha_{p m} & =\arctan \frac{\left(x_{m}-x_{p}\right)^{2}+\left(y_{m}-y_{p}\right)^{2}}{z_{p}-z_{c}} ; \\
\alpha_{p e} & =\arctan \frac{\left(x_{e}-x_{p}\right)^{2}+\left(y_{e}-y_{p}\right)^{2}}{z_{p}-z_{c}}
\end{aligned}
$$

Moreover, the movement of the RGB-D sensor is obtained considering that the cart is also moving. Thus, the objective is that $\alpha_{c}$ occupies the place of the angle opposite to $\alpha_{p}$, that is, $-\alpha_{p}$. Again, there are two rotation possibilities for the RGB-D sensor to reach the desired point:

$$
\begin{gathered}
\Delta \alpha_{1}=-\alpha_{p}-\alpha_{c} \\
\Delta \alpha_{2}=2 \pi+\alpha_{p}+\alpha_{c}
\end{gathered}
$$



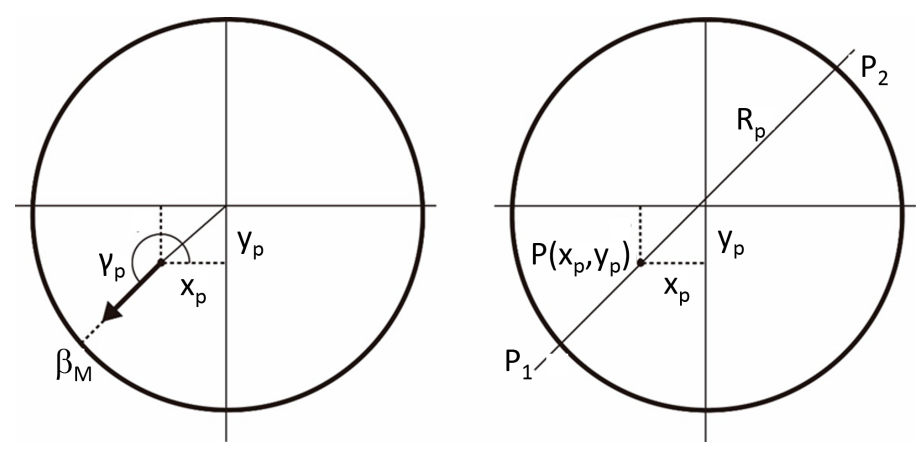

Fig. 3. Angular positions of the carts.

\subsection{Angular Positions of the Carts}

The destination of the carts are defined through their current positions, the patient's position with respect to the centre of the circle, and the observed patient's direction. The new RGB-D sensor position to observe the patient's direction is the angular position of the circle that intersects with the imaginary line that passes through the direction of the patient with origin in his/her current position.

By means of this approach, the two cut points of the line passing through the position of the patient are obtained geometrically, taking the patient as the direction (see Fig. 3). To obtain line $R_{p}$, the position of the patient $P=\left(x_{p}, y_{p}\right)$ and the patient's direction $v_{p}=\left(x_{v}, y_{v}\right)=\left(\cos \gamma_{p}, \sin \gamma_{p}\right)$ are used, obtaining

$$
\frac{x-x_{p}}{x_{v}}=\frac{y-y_{p}}{y_{v}}
$$

being $x$ and $y$ the coordinates of the different points that belong to line $R_{p}$. On the other hand, the parametrisation of the circular path of radius $R$ is defined as

$$
x^{2}+y^{2}=R^{2}
$$

Operating with expressions (7) and (8), we get

$$
\left(m^{2}+1\right) y^{2}+2 m n y+\left(n^{2}-R^{2}\right)=0
$$

where $m=\frac{x_{v}}{y_{v}}$ and $n=x_{p}-\frac{y_{p}}{y_{v}} \cdot x_{v}$, resulting in cut points $P_{1}=\left(x_{1}, y_{1}\right)$ and $P_{2}=\left(x_{2}, y_{2}\right)$

$$
\begin{aligned}
& y_{1}=\frac{-2 m n+\sqrt{4 m^{2} n^{2}-4\left(m^{2}+1\right)\left(n^{2}-R^{2}\right)}}{2\left(m^{2}+1\right)} ; \quad x_{1}=\left(y_{1}-y_{p}\right) \cdot m+x_{p} \\
& y_{2}=\frac{-2 m n-\sqrt{4 m^{2} n^{2}-4\left(m^{2}+1\right)\left(n^{2}-R^{2}\right)}}{2\left(m^{2}+1\right)} ; \quad x_{2}=\left(y_{2}-y_{p}\right) \cdot m+x_{p}
\end{aligned}
$$


In order to obtain which point is correct, the parametric equation of line $R_{p}$ with the value of parameter $t=\frac{x_{1}-x_{p}}{x_{v}}=\frac{y_{1}-y_{p}}{y_{v}}$ is calculated by substituting in said equation the value of the first calculated point. If the value of parameter $t$ is positive, then point $P_{1}$ is the cart's destination point. If it is negative, then the cart must move to $P_{2}$.

The next step is using parameter $\beta_{m}=\arctan \frac{y}{x}$ to convert that point of the circumference into an angular position around the circle. Once we have obtained the point to be addressed, the cart movement has two possible solutions. The first one is $\Delta \beta_{1}=\beta_{m}-\beta_{c}$. The second one depends on the sign of the first, since it corresponds to the opposite direction. If $\Delta \beta_{1}$ corresponds to a clockwise turn, we have $\Delta \beta_{2}=2 \pi+\beta_{m}-\beta_{c}$. If the turn is counter-clockwise, we have $\Delta \beta_{2}=-2 \pi+\beta_{m}-\beta_{c}$. Next, the displacements $\Delta \beta$ of both carts are summed up and the role of each cart is selected as a function of said displacements. The movement function of the master cart is

$$
M_{C M}=\left(\min \left(\left|\Delta \beta_{1}\right|,\left|\Delta \beta_{2}\right|\right), \min \left(\left|\Delta \alpha_{1}\right|,\left|\Delta \alpha_{2}\right|\right), \min \left(\left|\Delta \gamma_{1}\right|,\left|\Delta \gamma_{2}\right|\right)\right)
$$

A similar mathematical development is carried out for the slave cart.

\subsection{Selection of the Carts' Roles}

The selection of each cart's role is determined according to the following criteria:

i. When the carts' destination points to cover the patient's position have been calculated, firstly the cart closest to the main vector becomes the master. The other cart is assigned the role of slave. However, this may change, as the total route made by both carts is priority when choosing the roles.

ii. Any collision between the carts must be avoided. For this purpose, it is checked that the route used by one cart is not interspersed with the other, avoiding such possible route.

iii. If both carts are at the same distance from point $\beta_{M}$, the cart closest to point $\beta_{E}$ becomes the slave.

iv. If both points are at the same distance from the two carts, the one whose speed in the direction of the point is larger is the one that takes the role of master.

v. And, if both carts have the same speed or are quiet, cart 1 will be the master and cart 2 will be the slave.

To explain these situations, two examples are analysed (see Fig. 4), where it is determined which of the two carts should go to each position according to the destination. In example (a) of the figure, the distribution of roles is very simple according to the first rule described. Cart 1 takes the role of master and moves to position $\beta_{M}$ since it is the closest one. On the other hand, cart 2 takes the slave role and moves to position $\beta_{E}$. In example (b), the same assignment of roles is done: cart 1 would be the master and go to point $\beta_{M}$, while cart B would take the role of slave and go to point $\beta_{E}$. However, the algorithm foresees a collision 

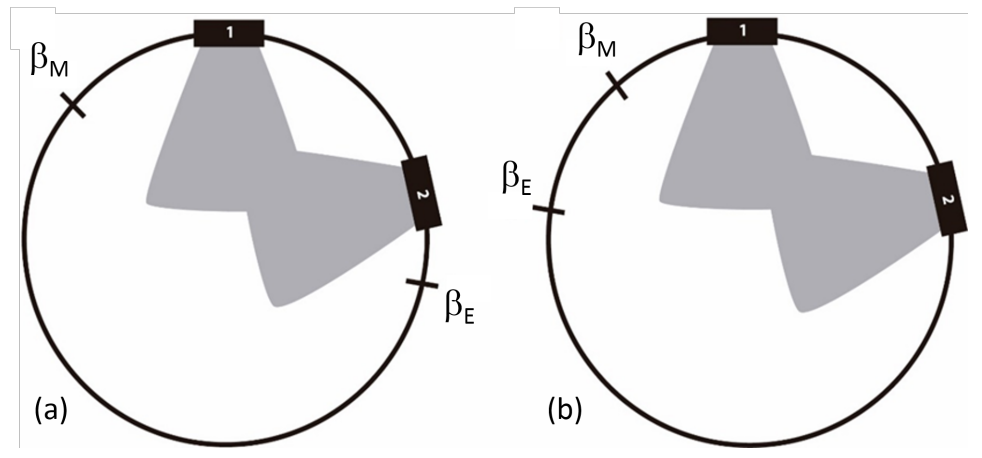

Fig. 4. Examples of role distribution.

of both carts, and immediately changes the trajectory of cart 2 to move to point $\beta_{E}$, which is at least slightly optimal. To avoid such situation, a change of roles is convenient, taking cart 1 the role of slave and cart 2 the role of master. In this way, the total journey made by both carts is much smaller and optimal.

\section{Study Case}

A study case presented to validate the functioning of the advanced trajectory generator. The complete tracking of a patient whose position has moved with respect to the centre of the circular rail is performed. Fig. 5a shows the position of the carts and the patient at the initial simulation instant. Fig. 5b illustrates the final positions reached, where it can be seen how the carts have reached the desired positions, just as the RGB-D sensor has successfully rotated to point directly to the patient.

The trajectory generator selects the shortest route and gives the role of master to cart $\mathrm{Q}$ and that of slave to $\mathrm{S}$. In addition, we compare the values calculated by the algorithm and the final route to check if the system has selected the shortest route. Table 1 collects the aforementioned data. As can be verified, the system selects the shortest path between the possible routes (clockwise or counter-clockwise). This is, the route in counter-clockwise direction for cart $\mathrm{Q}$ and the clockwise for cart S, respectively.

Table 1. Distance according to path.

\begin{tabular}{lrr}
\hline Data (rad) & Cart Q & Cart S \\
\hline Path clockwise & -5.1706 & -1.0147 \\
Path counter-clockwise & 1.1126 & 5.2685 \\
Path selected & 1.1126 & -1.0147 \\
\hline
\end{tabular}



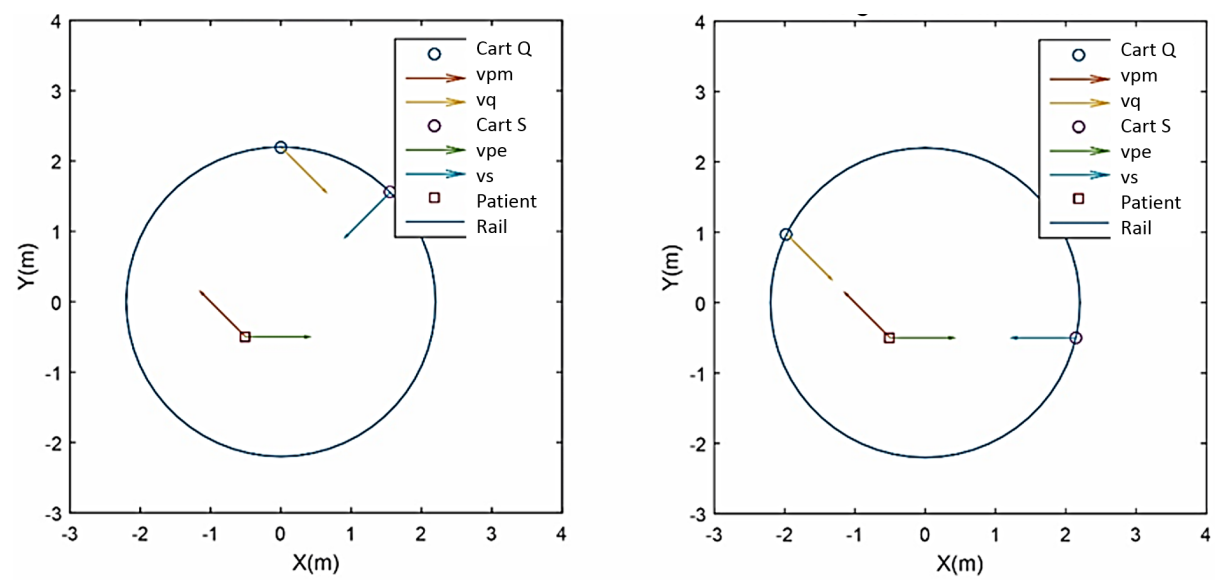

Fig. 5. Positioning graphs of the study case. Left: start positions. Right: final positions.

Besides, it is checked that the system has correctly selected the roles so that the total distance travelled by both carts is the minimum (see Table 2). Since cart Q was selected as master and cart S as slave, it is verified that the trajectory generator has correctly selected the shortest distance travelled by both carts.

Table 2. Distance according to role.

\begin{tabular}{lcc}
\hline Data $(\mathrm{rad})$ & $\mathrm{Q}$ master, S slave Q slave, S master \\
\hline Distance Q & 1.1126 & -1.8980 \\
Distance S & -1.0147 & 1.8980 \\
Total distance & 2.1273 & 3.796 \\
\hline
\end{tabular}

The next check is related to the tilt of the RGB-D sensor. The data shown in Table 2 throw the graphs shown in Fig. 6 . Since the cart's turn is now considered, the tilt angle of the RGB-D sensor must be the opposite of that direction. As can be seen in the graph, the direction of cart $\mathrm{Q}$ is tilted until it takes the angle opposite to the patient's master direction. In the same way, cart direction $\mathrm{S}$ is tilted to observe the slave vector of the patient.

As with positioning, it is checked that the system is able to select the shortest route. The data obtained for the study are included in Table 3. Finally, the pan angle that the RGB-D sensor takes to focus on the patient must be checked in the same direction but in the opposite sense. As can be seen from the graph, the vector of cart $Q$ tilts until it points directly to the patient's master vector. In the same way, cart $\mathrm{S}$ vector is tilted to observe the slave vector of the patient.

It is also observed that the system is able to select the shortest route. Table 4 shows that the system has correctly selected the route to travel the smallest 

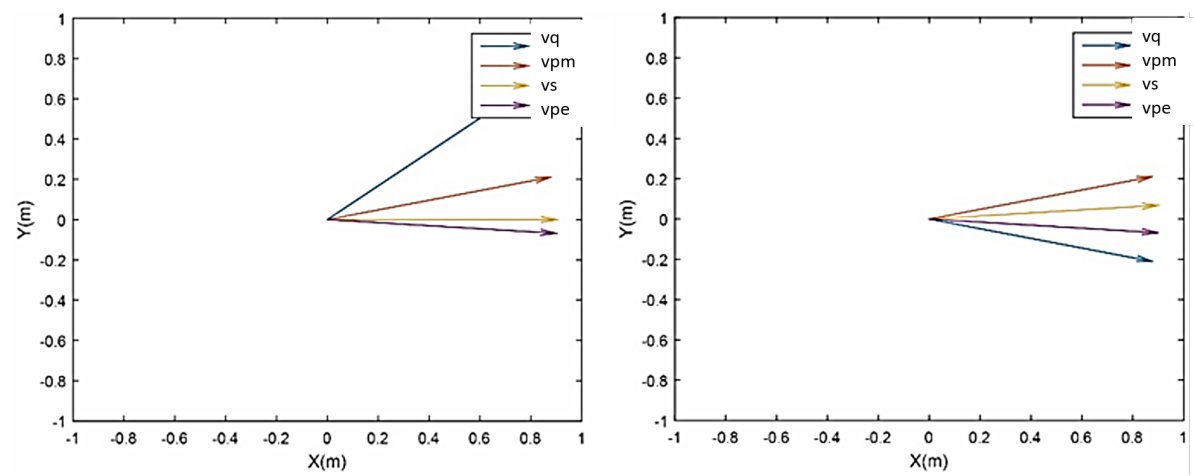

Fig. 6. Tilt graphs of the study case. Left: start positions. Right: final positions.

Table 3. Tilt according to path.

\begin{tabular}{lrr}
\hline Data (rad) & Cart Q & Cart S \\
\hline Path clockwise & -0.9337 & -6.2076 \\
Path counter-clockwise & 5.3495 & 0.0755 \\
Path selected & -0.933 & 0.0755 \\
\hline
\end{tabular}

possible path. In this case, routes are counter-clockwise and clockwise for carts $\mathrm{Q}$ and $\mathrm{S}$, respectively.

Table 4. Pan according to path.

\begin{tabular}{lrr}
\hline Data (rad) & Cart Q & Cart S \\
\hline Path clockwise & -1.1126 & 6.0539 \\
Path counter-clockwise & 5.1706 & 0.2293 \\
Path selected & -1.1126 & 0.2293 \\
\hline
\end{tabular}

The final displacement functions of carts $\mathrm{Q}$ and $\mathrm{S}$ are called $M_{C Q}$ and $M_{C S}$, respectively:

$$
\begin{gathered}
M_{C Q}(\beta, \alpha, \gamma)=(2.1271,-0.9337,-1.1126) \mathrm{rad} \\
M_{C S}(\beta, \alpha, \gamma)=(3.7960,-0.0755,0.2293) \mathrm{rad}
\end{gathered}
$$

\section{Conclusions}

This paper has presented an advanced trajectory generator for the motion of two carts with an RGB-D sensor aboard on a motorised circular rail. A continuous monitoring of the facial emotion expressed by the patient and the performed physical rehabilitation exercises is the final objective of the described system. 
This will be a future tool for evaluation of the proper physical exercises by calculating possible deviations of the optimal gestures, and, at the same, the degree of comfort of the patient during rehabilitation through the emotions felt. This is why, the paper has introduced the description of the displacements of both carts and the positioning of the tilt and pan angles of each RGB-D sensor to track the face and the body of the patient from the best viewpoints.

The paper has also described a study case that demonstrates that the proposed advanced trajectory generator offers enough security to ensure that both carts never collide during their tracking of the human undergoing a rehabilitation program.

\section{Acknowledgements}

This work was partially supported by Ministerio de Ciencia, Innovación y Universidades, Agencia Estatal de Investigación (AEI) / European Regional Development Fund (FEDER, UE) under DPI2016-80894-R grant.

\section{References}

1. Tian, G., Liu, L., Ri, J.H., Liu, Y.: ObjectFusion: An object detection and segmentation framework with RGB-D SLAM and convolutional neural networks. Author links open overlay panel bYiranSuna

2. Freitas, D., Gama, A. Da, Figueiredo, L., Chaves, T., Marques-Oliveira, D., Teichrieb, V., Araújo, C.: Development and evaluation of a Kinect based motor rehabilitation game. Simposio Brasileiro de Jogos e Entretenimento Digital, 144-153 (2012)

3. Chang, Y., Chen, S., Huang, J.: A Kinect-based system for physical rehabilitation: a pilot study for young adults with motor disabilities. Research in Developmental Disabilities, 326, 2566-2570 (2011)

4. López-Valles, J.M., Fernández, M.A., Fernández-Caballero, A: Stereovision depth analysis by two-dimensional motion charge memories. Pattern Recognition Letters 28(1), 20-30 (2007)

5. Oliver, M., Montero, F., Molina, J.P., González, P., Fernández-Caballero, A.: Multi-camera systems for rehabilitation therapies: a study of the precision of Microsoft Kinect sensors. Frontiers of Information Technology \& Electronic Engineering 17(4), 348-364 (2016)

6. Oliver, M., Montero, F., Fernández-Caballero, A., González, P., Molina, J.P.: RGB$\mathrm{D}$ assistive technologies for acquired brain injury: description and assessment of user experience. Expert Systems 32(3), 370-380 (2015)

7. Castillo, J.C., Fernández-Caballero, A., Castro-González, Á., Salichs, M.A., López, M.T.: A framework for recognizing and regulating emotions in the elderly. Lecture Notes in Computer Science 8868, 320-327 (2014)

8. Lozano-Monasor, E., López, M.T., Fernández-Caballero, A., Vigo-Bustos, F.: Facial expression recognition from webcam based on active shape models and support vector machines. Lecture Notes in Computer Science 8868, 147-154 (2014)

9. Wang, P., Li, W., Ogunbona, P., Wan, J., Escalera, S.: RGB-D-based human motion recognition with deep learning: A survey. Computer Vision and Image Understanding 171, 118-139 (2018) 
10. Fernández-Caballero, A., López, M.T., Saiz-Valverde, S.: Dynamic stereoscopic selective visual attention (DSSVA): Integrating motion and shape with depth in video segmentation. Expert Systems with Applications 34(2), 1394-1402 (2008)

11. Castillo, J.C., Fernández-Caballero, A., Serrano-Cuerda, J., López, M.T., Martínez-Rodrigo, A.: Smart environment architecture for robust people detection by infrared and visible video fusion. Journal of Ambient Intelligence and Humanized Computing 8(2), 223-237 (2017)

12. Fernández-Caballero, A., López, M.T., Serrano-Cuerda, J.: Thermal-infrared pedestrian ROI extraction through thermal and motion information fusion. Sensors 14(4), 6666-6676 (2014)

13. Moreno-Garcia, J., Rodriguez-Benitez, L., Fernández-Caballero, A., López, M.T.: Video sequence motion tracking by fuzzification techniques. Applied Soft Computing 10(1), 318-331 (2010)

14. Florea, A.G., Buiu, C.: A distributed approach to the control of multi-robot systems using XP colonies. Integrated Computer-Aided Engineering 25(1), 15-29 (2018)

15. Morales, R., Chocoteco, J., Feliu, V., Sira-Ramírez, H.: Obstacle surpassing and posture control of a stair-climbing robotic mechanism. Control Engineering Practice 21, 604-621 (2013)

16. Morales, R., González, A., Feliu, V., Pintado, P.: Environment adaptation of a new staircase climbing wheelchair. Autonomous Robots 23, 275-292 (2007)

17. Panwar, R., Sukavanam, N.: Trajectory tracking using artificial neural network for stable human-like gait with upper body motion. Neural Computing \& Applications (2018).

18. Almansa-Valverde, S., Castillo, J.C., Fernández-Caballero, A.: Mobile robot map building from time-of-flight camera. Expert Systems with Applications 39(10), 8835-8843 (2012)

19. Gascueña, J.M., Fernández-Caballero, A.: Agent-oriented modeling and development of a person-following mobile robot. Expert Systems with Applications 38(4), 4280-4290 (2011)

20. Morales, R., Somolinos, J.A., Fernández-Caballero, A., Ferraresi, C.: Rehabilitation robotics and systems. Journal of Healthcare Engineering 2018, 5370127 (2018)

21. Chocoteco, J., Morales, R., Feliu, V.: Enhancing the trajectory generation of a stair-climbing mobility system. Sensors 17(1), 1-31 (2017)

22. Chocoteco, J., Morales, R., Feliu, V., Sánchez, L.: Trajectory planning for a stairclimbing mobility system using laser distance sensors. IEEE Systems Journal 10(3), 944-956 (2016)

23. Benito-Picazo, J., Domínguez, E., Palomo, E.J., López-Rubio, E., Ortiz-deLazcano-Lobato, J.M.: Motion detection with low cost hardware for PTZ cameras. Integrated Computer-Aided Engineering 26(1), 21-36 (2019)

24. Panduro, R., Oliver, M., Morales, R., González, P., Fernández-Caballero, A.: Motorized multi-camera slider for precise monitoring of physical rehabilitation. Lecture Notes in Computer Science 10070, 21-27 (2016)

25. Mkhitaryan, A., Burschka, D.: RGB-D sensor data correction and enhancement by introduction of an additional RGB view. Proceedings of the IEEE/RSJ International Conference on Intelligent Robots and Systems, 1077-1083 (2013) 